\title{
BOREL FUNCTIONS OF BOUNDED CLASS
}

BY

D. H. FREMLIN, R. W. HANSELL AND H. J. K. JUNNILA

\begin{abstract}
ABSTRACr. Let $X$ and $Y$ be metric spaces and $f: X \rightarrow Y$ a Borel measurable function. Does $f$ have to be of bounded class, i.e. are the sets $f^{-1}[H]$, for open $H \subseteq Y$, of bounded Baire class in $X$ ? This is an old problem of A. H. Stone. Positive answers have been given under a variety of extra hypotheses and special axioms. Here we show that (i) unless something similar to a measurable cardinal exists, then $f$ is of bounded class and (ii) if $f$ is actually a Borel isomorphism, then $f\left(\right.$ and $\left.f^{-1}\right)$ are of bounded class.
\end{abstract}

0. Introduction. Our main aim is to prove the results announced above, but we find that minor changes of wording enable us to deal at the same time with some other problems which have been raised. To provide a framework for our discussion, we start with a definition. Let $Q$ be a family of subsets of a set $X$. For ordinals $\xi$, define $\mathscr{B}_{\xi}(Q)$ inductively by writing

$$
\begin{aligned}
\mathscr{B}_{\xi}(\mathscr{Q}) & =\left\{\bigcup_{n \in \mathbf{N}} A_{n}: \forall n \in \mathbf{N}, A_{n} \in \bigcup_{\eta<\xi} \mathscr{B}_{\eta}(\mathscr{Q})\right\} \cup Q, \\
\mathscr{B}_{\xi+1}(\mathscr{Q}) & =\left\{\bigcap_{n \in \mathbf{N}} A_{n}: \forall n \in \mathbf{N}, A_{n} \in \mathscr{B}_{\xi}(\mathbb{Q})\right\}
\end{aligned}
$$

for every even ordinal $\xi$ (counting limit ordinals as even). Then the $\mathscr{B}_{\xi}(\mathcal{Q})$ form an increasing family which stops growing at or before the first uncountable ordinal $\omega_{1}$. We write $\mathscr{B}(\mathbb{Q})$ for $\Re_{\omega_{1}}(\mathscr{Q})=\cup_{\xi<\omega_{1}} \Re_{\xi}(\mathscr{Q})$. Now, if $Y$ is a topological space and $R \subseteq X \times Y$ is a relation, we say that $R$ is lower $\Re(\mathscr{Q})$-measurable if $R^{-1}[H]=\{t$ : $\exists u \in H,(t, u) \in R\} \in \mathscr{B}(Q)$ for every open $H \subseteq Y$, and that $R$ is of bounded class (with respect to $Q$ ) if there is a $\xi<\omega_{1}$ such that $R^{-1}[H] \in \mathscr{B}_{\xi}(\mathbb{Q})$ for every open $H \subseteq Y$. We shall be concerned with questions of the form: under what conditions, given that $R$ is measurable, can we conclude that it is of bounded class?

An elementary case presents itself at once. If $Y$ is second-countable, then any $R$ will be of bounded class for any $X$ and $\mathcal{Q}$ (cf., for example, [10, §31, II, Theorem 1]). In other cases (as in Proposition 8 below) we find that an analysis of certain families of subsets of $X$ is sufficient. Let us say that a family $\mathcal{E} \subseteq \mathscr{P} X$ is $\mathcal{H}$-additive, where $\mathcal{H} \subseteq \mathcal{P} X$, if $\cup \mathcal{E}^{\prime} \in \mathcal{H}$ for every $\mathcal{E}^{\prime} \subseteq \mathcal{E}$. Then we shall need to ask: under what circumstances, given that $\mathscr{E}$ is $\mathscr{B}(\mathscr{Q})$-additive, can we conclude that it is $\mathscr{B}_{\xi}(\mathbb{Q})$-additive for some $\xi<\omega_{1}$ ? When it is, we shall say that $\mathcal{E}$ is boundedly $\mathscr{B}(\mathscr{Q})$-additive. This corresponds to the previous question when $Y$ is discrete.

Received by the editors August 11, 1981 and, in revised form, August 13, 1982.

1980 Mathematics Subject Classification. Primary 54H05; Secondary 04A15.

(C) 1983 American Mathematical Society 0002-9947/82/0000-0891/\$04.50 
The basis of this paper is Theorem 9 below, in which we discuss (in effect) the special case in which $\mathscr{B}(\mathscr{Q})=\mathscr{P} X$. The effect of this theorem is to show, first, that if there is a Borel measurable function between metric spaces which is not of bounded class, then something very extraordinary is going on; and second, that the cardinal $\omega_{1}$, which one would expect to be the villain of the piece, is far too small and must be entirely innocent.

We begin with statements of the more or less well-known facts on which we shall rely $(\S \S 2-8)$; then give our principal Theorem 9 and its corollaries, many of which are known ( $\S 10-17)$. Our results are summarized in Theorem 16. This relies on Theorem 12, which seems to be of independent interest, though it is really a restatement of known results. Our other main result (Theorem 19) solves Stone's problem for Borel isomorphisms; we mention two corollaries (\$§20-21). We show that Theorem 9 at least cannot be collapsed by a simple argument (Proposition 22), and conclude with a discussion of problems still outstanding (\$23).

We should like to thank the referee for some helpful remarks on our proofs.

1. Notation. We use the following terminology. A collection $\mathcal{E}$ of sets is point-finite if $\{E: t \in E \in \mathcal{E}\}$ is finite for every $t$. If $R$ is a relation (i.e. a class of ordered pairs) we write

$$
\begin{aligned}
R[A] & =\{u: \exists t \in A,(t, u) \in R\}, \\
R^{-1}[B] & =\{t: \exists u \in B,(t, u) \in R\}
\end{aligned}
$$

for any $A, B$. If $X$ is a set equipped with an equivalence relation, we write $t^{\bullet}$ for the equivalence class of any $t \in X$; in particular, if $\Sigma$ is an algebra of sets and 9 is an ideal of $\Sigma$, we write $E^{\bullet} \in \Sigma / 9$ for the canonical image of any $E \in \Sigma$.

2. Properties of metric spaces. If $\mathfrak{I}$ is the topology of a metric space $X$, then the $\mathscr{B}_{\xi}(\mathfrak{I})$ in our notation are just the $\mathbf{G}_{\xi}$ of $[\mathbf{1 0}, \S 30, \mathrm{II}]$, and $\mathscr{B}(\mathfrak{I})$ is the family of Borel sets. On occasion we shall write $\mathscr{B}_{\xi}(X), \Re_{(}(X)$ in place of $\mathscr{B}_{\xi}(\mathfrak{I})$ and $\mathscr{B}(\mathfrak{I})$, if there seems no room for confusion.

In any topological space $X$, let us say (following [10]) that $\mathcal{E} \subseteq \mathcal{P} X$ is discrete if every point of $X$ has a neighbourhood meeting at most one member of $\mathcal{E}$; $\sigma$-discrete if it is expressible as $\cup_{n \in \mathbf{N}} \mathcal{E}_{n}$ where each $\mathcal{E}_{n}$ is discrete; and $\sigma$-discretely decomposable if there is a family $\left\langle C_{E}^{n}\right\rangle_{E \in \mathcal{E}, n \in \mathbf{N}}$ such that $E=\cup_{n \in \mathbf{N}} C_{E}^{n}$ for each $E \in \mathcal{E}$ and $\left\langle C_{E}^{n}\right\rangle_{E \in \mathcal{E}}$ is discrete (i.e. $\left\langle C_{E}^{n}\right\rangle_{E \in \mathcal{E}}$ is disjoint and $\left\{C_{E}^{n}: E \in \mathcal{E}\right\}$ is discrete) for each $n \in \mathbf{N}$.

We shall make repeated use of the following facts.

3. THEOREM. (a) Every metric space has a o-discrete base for its topology $[10, \S 21, X V I$, Corollary 1a].

(b) Let $X$ be a metric space, $\mathcal{E}$ a point-finite $\mathscr{G}_{\eta}(X)$-additive family of subsets of $X$, and $\left\langle F_{E}\right\rangle_{E \in \mathcal{E}}$ any family in $\mathscr{B}_{\xi}(X)$, where $\eta, \xi<\omega_{1}$. Then $\cup_{E \in \mathcal{E}}\left(E \cap F_{E}\right) \in$ $\Re_{\eta^{\prime}+\xi}(X)$, where $\eta^{\prime}=\eta$ if $\eta$ is even, $\eta+1$ if $\eta$ is odd $[6$, Theorem 3.6].

4. Algebras generated by rectangles. Let $X$ and $Y$ be sets, $\Sigma$ and $T$ algebras of subsets of $X$ and $Y$ respectively. We write $\Sigma \hat{\otimes}_{\sigma} T$ for the $\sigma$-algebra of subsets of $X \times Y$ generated by $\{E \times F: E \in \Sigma, F \in T\}$. (In the notation of $\S 1$, this is just 
$\Re(\{E \times F: E \in \Sigma, F \in T\}$.) We shall be much concerned with cases in which $\mathscr{P}(X \times Y)=\mathscr{P} X \hat{\otimes}_{\sigma} \mathscr{P} Y$. We describe one set of circumstances under which this occurs in Theorem 12 below. Apart from this, we need to know that if $X$ and $Y$ both have cardinal $\leqslant \omega_{1}$, then $\mathscr{P}(X \times Y)=\mathscr{P} X \hat{\otimes}_{\sigma} \mathscr{P} Y[9$, Theorem 12.5(ii); 14, Theorem $1]$.

5. A combinatorial lemma. Let $X$ be a set, and $\mathscr{E} \subseteq \mathcal{P} X$ a point-finite family of sets. If $\#(\mathcal{E})=\kappa>\omega$, then there is $a Z \subseteq X$ and an $\mathcal{E}_{1} \subseteq \mathcal{E}$ such that $\#(Z)=\kappa$, each member of $Z$ belongs to just one member of $\mathcal{E}_{1}$, and each member of $\mathcal{E}_{1}$ contains just one member of $Z$.

Proof. Enumerate $\mathcal{E} \backslash\{\varnothing\}$ as $\left\langle E_{\xi}\right\rangle_{\xi<\kappa}$, and choose $t_{\xi} \in E_{\xi}$ for each $\xi<\kappa$. Set $f(\xi)=\left\{\eta: \eta<\kappa, \eta \neq \xi, t_{\xi} \in E_{\eta}\right\}$; then each $f(\xi)$ is a finite subset of $\kappa$ not containing $\xi$. By Hajnal's theorem [2, Theorem 10.14], there is an $A \subseteq \kappa$ such that $\#(A)=\kappa$ and $\eta \notin f(\xi)$ whenever $\xi, \eta \in A$. Set $Z=\left\{t_{\xi}: \xi \in A\right\}, \mathcal{E}_{1}=\left\{E_{\xi}: \xi \in A\right\}$.

6. Ideals in $\mathscr{P} X$. Let $X$ be a set, $\mathscr{G}$ an ideal for $\mathscr{P} X, \kappa$ a cardinal. We say tht $\mathscr{G}$ is a $\kappa$-ideal if the union of $\kappa$ members of 9 always belong to $G$, and that $G$ is $\kappa$-saturated if there is no disjoint family of cardinal $\kappa$ in the Boolean algebra $\mathscr{P} X / \mathcal{G}$. Note that if $\mathscr{G}$ is a $\kappa$-ideal, it is $\kappa^{+}$-saturated iff there is no disjoint family of cardinal $\kappa^{+}$in $\mathscr{P} X \backslash \mathscr{9}$. We write $\kappa_{d}$ for the least cardinal of any set $X$ such that $\mathscr{P} X$ has an $\omega_{1}$-saturated $\sigma$-ideal $\mathscr{G}$ for which $\mathscr{P} X / \mathscr{G}$ is atomless and $X \notin \mathscr{G}$, if such a set exists; if there is no such $X$, we write $\kappa_{d}=\infty$.

The standard text for such questions is [15]. The situation is: it is certainly consistent to suppose that $\kappa_{d}=\infty$ (for instance, the continuum hypothesis implies this). It is not so surely consistent to suppose that $\kappa_{d}<\infty$, but it appears likely to be; this is equiconsistent with the existence of a nonprincipal ultrafilter closed under countable intersections (see [15]). Martin's Axiom does not affect the issue [11, p. 163]. But we do have the following results.

7. LEMMA. (a) If $\mathscr{G}$ is a $\kappa^{+}$-saturated $\kappa$-ideal of $\mathscr{P} X$ such that $\mathscr{P} X / \mathcal{G}$ is atomless, then $X$ is expressible as the union of not more than $2^{\kappa}$ members of 9 .

(b) If $\kappa_{d}<\infty$ then $\kappa_{d} \leqslant \mathrm{c}$; consequently there are unthinkably many cardinals $\leqslant \mathrm{c}$.

(c) If $\kappa_{d}<\infty$ and $\mathcal{G}$ is an $\omega_{1}$-saturated $\sigma$-ideal of $\mathscr{P} X$, then the union of fewer than $\kappa_{d}$ members of 9 belongs to 9 .

(d) If $\mathcal{G}$ is an $\omega_{1}$-saturated $\sigma$-ideal of $\mathcal{P} X$, then the union of $\omega_{1}$ members of 9 belongs to 9 .

Proof. (a) If $X \in \mathscr{Q}$, this is trivial; suppose that $X \notin \mathscr{G}$. For each $A \in \mathcal{P} X \backslash \mathscr{q}$, choose a set $R(A) \subseteq A$ such that neither $R(A)$ nor $A \backslash R(A)$ belongs to 9 ; this is possible because, by hypothesis, $A^{*}$ is not an atom in $\mathscr{P} X / \mathscr{G}$. Construct $\mathcal{T}_{\xi} \subseteq \mathscr{P} X$, for ordinals $\xi<\kappa^{+}$, inductively, as follows. $\mathscr{T}_{0}=\{X\}$. Given that $\mathscr{T}_{\xi}$ is a disjoint family in $\mathscr{P} X \backslash 9$, let $\mathscr{T}_{\xi+1}$ be

$$
\left\{R(A): A \in \mathcal{T}_{\xi}\right\} \cup\left\{A \backslash R(A): A \in \mathcal{T}_{\xi}\right\} ;
$$

then $\mathscr{T}_{\xi+1}$ is a disjoint family in $\mathscr{P} X \backslash \mathscr{T}$ which refines $\mathcal{T}_{\xi}$ (i.e. for every $B \in \mathcal{T}_{\xi+1}$ there is an $A \in \mathcal{T}_{\xi}$ such that $B \subseteq A$ ). For limit ordinals $\xi>0$, given that $\left\langle\mathcal{T}_{\eta}\right\rangle_{\eta<\xi}$ is a 
family of disjoint families in $\mathscr{P} X \backslash \mathscr{q}$ such that $\mathcal{T}_{\eta}$ refines $\mathcal{\sigma}_{\zeta}$ whenever $\zeta \leqslant \eta<\xi$, let $\mathcal{T}_{\xi}$ be a maximal disjoint family in $\mathscr{P} X \backslash 9$ such that $\mathcal{T}_{\xi}$ refines $\mathcal{T}_{\eta}$ for every $\eta<\xi$.

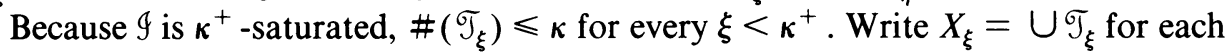
$\xi<\kappa^{+}$. Then $\cap_{\xi<\kappa^{+}} X_{\xi}=\varnothing$. For suppose, if possible, that $t \in \bigcap_{\xi<\kappa^{+}} X_{\xi}$. For each $\xi<\kappa^{+}$let $A_{\xi}$ be the member of $\mathcal{T}_{\xi}$ containing $t$. Then $A_{\xi} \subseteq A_{\eta}$ whenever $\eta \leqslant \xi<\kappa^{+}$, and $\left\langle A_{\xi} \backslash A_{\xi+1}\right\rangle_{\xi<\kappa^{+}}$is a disjoint family in $\mathscr{P} X \backslash 9$, which is impossible.

For each nonzero limit ordinal $\xi<\kappa^{+}$, let

$$
Y_{\xi}=\bigcap_{\eta<\xi} X_{\eta} \backslash X_{\xi}
$$

On $Y_{\xi}$ each $\mathcal{T}_{\eta}$, for $\eta<\xi$, defines a partition; as $\mathcal{T}_{\eta}$ refines $\mathcal{T}_{\zeta}$ for $\zeta \leqslant \eta<\xi$, the $\mathcal{T}_{\eta}$ together define a partition $\mathscr{2}_{\xi}$ say. Because $Y_{\xi} \cap X_{\xi}=\varnothing$, and $\sigma_{\xi}$ was chosen to be maximal, every element of $\mathscr{Q}_{\xi}$ must belong to $\mathscr{~}$. Also, each $\mathcal{T}_{\eta}$ has cardinal $\leqslant \kappa$; so $\#\left(\cup_{\eta<\xi} \sigma_{\eta}\right) \leqslant \kappa$, and $\#\left(\mathscr{Q}_{\xi}\right) \leqslant 2^{\kappa}$.

Thus each $Y_{\xi}$ is covered by $2^{\kappa}$ or fewer members of 9 . But $X$ is the union of the $Y_{\xi}$, because $X_{0}=X$ and $X_{\xi+1}=X_{\xi}$ for every $\xi$. So $X$ is also covered by $2^{\kappa}$ or fewer members of 9 (because $\kappa^{+} \leqslant 2^{\kappa}$ ).

(b) Setting $\kappa=\omega$ in (a), we see that if $\kappa_{d}<\infty$ there is a set $X$ and a proper $\omega_{1}$-saturated $\sigma$-ideal $\mathscr{G}$ of $\mathscr{P} X$ such that $X$ is the union of $\mathfrak{c}$ or fewer members of 9 . Express $X$ as $\cup_{\xi<\lambda} I_{\xi}$ where $\lambda \leqslant c$ and $\left\langle I_{\xi}\right\rangle_{\xi<\lambda}$ is a disjoint family in $\mathcal{G}$. Let $g$ be $\{A$ : $\left.A \subseteq \lambda, \cup_{\xi \in A} I_{\xi} \in 9\right\}$. Then $\mathscr{g}$ is a proper $\omega_{1}$-saturated $\sigma$-ideal of subsets of $\lambda$, which contains all singletons. But now $\mathscr{P} \lambda / \mathscr{G}$ must be atomless; for if $A \subseteq \lambda$ is such that $A^{*}$ is an atom in $\mathcal{P} \lambda / \mathcal{G}$, then $\{B: B \subseteq A, B \notin \mathcal{G}\}$ is a nonprincipal ultrafilter on $A$ which is closed under countable intersections; and because $\#(A) \leqslant \lambda \leqslant \mathfrak{c}$, this is impossible (Ulam's easy trick; see [3, Chapter 6, Theorem 1.2]). It follows that $\kappa_{d} \leqslant \lambda \leqslant c$.

Now [15, Theorem 1] shows that there are unthinkably many cardinals less than $\kappa_{d}$.

(c)-(d) Now suppose that $X$ is any set and 9 an $\omega_{1}$-saturated $\sigma$-ideal of subsets of $X$. Suppose that $\left\langle I_{\xi}\right\rangle_{\xi<\lambda}$ is a disjoint family in 9 such that $\cup_{\xi<\lambda} I_{\xi} \notin 9$. Passing to an ideal $\mathcal{G}$ of $\mathscr{P} \lambda$ as in (b) above, we see that either $\mathscr{P} \lambda / \mathcal{G}$ has an atom, so that $\lambda>c$, or it is atomless, so that $\lambda \geqslant \kappa_{d}$. In either case, if $\kappa_{d}<\infty, \lambda \geqslant \kappa_{d}$; proving (c). Finally, $\kappa_{d}$ is surely greater than $\omega_{1}([15$, Theorem 1]; the argument is contained in Exercise 1.9(3) of [3, Chapter 6]); so that in either case $\lambda>\omega_{1}$, proving (d).

REMARK. We do not need the full strength of 7(a) in this paper, but we have found uses for it elsewhere.

8. Proposition. Let $(X, \mathfrak{I})$ be a metrizable topological space.

(a) The following are equivalent: (i) every Borel measurable function from $X$ to a metric space $Y$ is of bounded class; (ii) every Borel-additive partition of $X$ is boundedly Borel-additive.

(b) The following are equivalent: (i) whenever $Y$ is a metric space and $R \subseteq X \times Y$ is a lower Borel measurable relation with compact vertical sections, then $R$ is of bounded class; (ii) every point-finite Borel-additive family of subsets of $X$ is boundedly Boreladditive. 
Proof. (a) (i) $\Rightarrow$ (ii). Trivial, considering discrete spaces $Y$.

(ii) $\Rightarrow$ (i). Assume (ii), and let $Y$ be a metric space, $f: X \rightarrow Y$ a Borel measurable function. Let $\mathscr{U}=\cup_{n \in N} \mathscr{Q}_{n}$ be a base for the topology of $Y$, where each $\mathscr{U}_{n}$ is discrete (Theorem 3(a) above). Now

$$
\mathcal{E}_{n}=\left\{f^{-1}[U]: U \in \mathcal{Q}_{n}\right\} \cup\left\{X \backslash f^{-1}\left[\cup \mathcal{Q}_{n}\right]\right\}
$$

is a Borel-aciditive partition of $X$, so is $\mathscr{B}_{\xi_{n}}(\mathfrak{I})$-additive for some $\xi_{n}<\omega_{1}$. Set $\xi=\sup _{n \in \mathbf{N}}\left(\xi_{n}+1\right)$; then

$$
f^{-1}[H]=\bigcup_{n \in \mathbf{N}} \bigcup\left\{f^{-1}[U]: U \in \mathscr{Q}_{n}, U \subseteq H\right\} \in \mathscr{P}_{\xi}(\mathfrak{I})
$$

for every open $H \subseteq Y$, and $f$ is of bounded class.

(b) (i) $\Rightarrow$ (ii). Again, nearly trivial; if (i) is true, and $\mathcal{E}$ is a point-finite Borel-additive family in $\mathcal{P} X$, then give $\mathcal{E}$ the discrete topology, and set $R=\{(t, E): t \in E \in$ $\mathcal{E}\} \subseteq X \times \mathcal{E}$. The vertical sections of $R$ are compact because $\mathcal{E}$ is point-finite. If $\mathcal{E}^{\prime} \subseteq \mathcal{E}$ then $R^{-1}\left[\mathcal{E}^{\prime}\right]=\cup \mathcal{E}^{\prime} \in \mathscr{B}(\mathfrak{T})$, so $R$ is lower Borel measurable. Accordingly it is of bounded Borel class, and there is a $\xi<\omega_{1}$ such that $\cup \mathcal{E}^{\prime}=R^{-1}\left[\mathcal{E}^{\prime}\right] \in \mathscr{B}_{\xi}(\mathfrak{I})$ for every $\mathcal{E}^{\prime} \subseteq \mathcal{E}$.

(ii) $\Rightarrow$ (i). Assume (ii), and let $Y$ be a metric space, $R \subseteq X \times Y$ a lower Borel measurable relation with compact vertical sections. Again let $\mathscr{Q}=\cup_{n \in \mathrm{N}} \mathscr{Q}_{n}$ be a base for the topology of $Y$ in which each $\mathcal{U}_{n}$ is discrete, and let $\varepsilon_{n}=\left\{R^{-1}[B]\right.$ : $\left.B \in \mathcal{Q}_{n}\right\}$. For each $t \in X,\{u:(t, u) \in R\}$ is compact, so can meet only finitely many members of $\mathscr{U}_{n}$; thus $\mathcal{E}_{n}$ is point-finite. Now we can argue as in (a) above.

REMARKS. In (b)(ii), we can replace the phrase "compact vertical sections" by

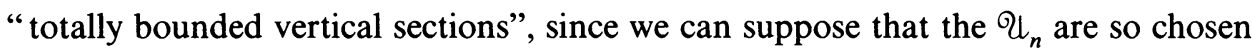
that the distance between two distinct members of $\mathscr{U}_{n}$ is always at least $2^{-n}$ (see $[10, \S 21$, XVI, Theorem 1]).

A recent result of B. S. Spahn shows that in fact (a) and (b) above are equivalent; see 23(f) below. We do not know whether every metric space satisfies these conditions; see Theorem 16 and $\$ 23$.

9. THEOREM. Let I be a set and $\left\langle\mathbb{Q}_{\xi}\right\rangle_{\xi<\omega_{1}}$ a family of subsets of $\mathscr{P} I$ such that if $\left\langle A_{n}\right\rangle_{n \in \mathrm{N}}$ is any sequence in $\cup_{\eta<\xi} \mathbb{Q}_{\eta}$, where $\xi$ is even, then $\cup_{n \in \mathrm{N}} A_{n} \in \mathbb{Q}_{\xi}$; if $\left\langle A_{n}\right\rangle_{n \in \mathrm{N}}$ is a decreasing sequence in $\mathbb{Q}_{\xi}$, where $\xi$ is even, then $\cap_{n \in \mathrm{N}} A_{n} \in \mathbb{Q}_{\xi+1}$; $\cup_{\xi<\omega_{1}} \mathbb{Q}_{\xi}=\mathscr{P} I$. Set $\mathscr{S}_{\xi}=\left\{A: A \subseteq I, \mathscr{P} A \subseteq \mathbb{Q}_{\xi}\right\}, \mathscr{G}=\cup_{\xi<\omega_{1}}{ }_{\xi}$. Then:

(a) If $\kappa$ is a cardinal such that $\mathscr{P}\left(\omega_{1} \times \kappa\right)=\mathscr{P} \omega_{1} \hat{\otimes}_{\sigma} \mathscr{P}_{\kappa}$, and $\left\langle A_{\xi}\right\rangle_{\xi<\kappa}$ is a point-finite family of subsets of $I$, then there is a $\zeta<\omega_{1}$ such that $\cup_{\xi \in C} A_{\xi} \in \mathbb{Q}_{\zeta}$ for every $C \subseteq \kappa$.

(b) If $\mathbb{Q}$ is any point-finite family of subsets of $I$, there is a $\zeta<\omega_{1}$ such that $Q \backslash q_{\zeta}$ is countable.

(c) $\mathscr{G}$ is an $\omega_{1}$-saturated $\sigma$-ideal of $\mathscr{P} I$ and $\mathscr{P} I / \mathscr{G}$ is atomless.

(d) There is $a \zeta<\omega_{1}$ such that $\mathscr{G}=g_{\zeta}$.

Proof. (a) Consider

$$
\mathscr{D}=\left\{D: D \subseteq \omega_{1} \times \kappa, \exists \zeta<\omega_{1}, \bigcup_{(\eta, \xi) \in D} A_{\xi} \in \mathbb{Q}_{\zeta} \forall \eta<\omega_{1}\right\} .
$$


This contains $B \times C$ for every $B \subseteq \omega_{1}, C \subseteq \kappa$ and is closed under countable unions and monotonic countable intersections (because $\left\langle A_{\xi}\right\rangle_{\xi<\kappa}$ is point-finite). So $\mathscr{D} \supseteq$ $\mathscr{P} \omega_{1} \hat{\otimes}_{\sigma} \mathscr{P}_{\kappa}=\mathscr{P}\left(\omega_{1} \times \kappa\right)$. Accordingly, if $\langle C(\eta)\rangle_{\eta<\omega_{1}}$ is any family of subsets of $\kappa$, then $D=\{(\eta, \xi): \xi \in C(\eta)\} \in \mathscr{D}$, and there is a $\zeta<\omega_{1}$ such that $\cup_{\xi \in C(\eta)} A_{\xi} \in \mathbb{Q}_{\zeta}$ for every $\eta<\omega_{1}$. It follows at once that there is a $\zeta<\omega_{1}$ such that $\bigcup_{\xi \in C} A_{\xi} \in \mathbb{G}_{\zeta}$ for every $C \subseteq \kappa$.

(b) For suppose, if possible, that there is no such $\zeta$. Then we could choose inductively $A_{\xi} \in \mathbb{Q} \backslash\left(\Phi_{\xi} \cup\left\{A_{\eta}: \eta<\xi\right\}\right)$ for every $\xi<\omega_{1}$. Now $\left\langle A_{\xi}\right\rangle_{\xi<\omega_{1}}$ is a pointfinite family and no $A_{\xi}$ belongs to $\Phi_{\xi}$. Choose $A_{\xi}^{\prime} \in \mathscr{P} A_{\xi} \backslash \mathbb{Q}_{\xi}$ for each $\xi<\omega_{1}$. Then $\left\langle A_{\xi}^{\prime}\right\rangle_{\xi<\omega_{1}}$ is a point-finite family. But we know that $\mathscr{P}\left(\omega_{1} \times \omega_{1}\right)=\mathscr{P} \omega_{1} \hat{\otimes}_{\sigma} \mathscr{P} \omega_{1}(\S 4$ above), so there must be a $\zeta<\omega_{1}$ such that $\cup_{\xi \in C} A_{\xi}^{\prime} \in \mathbb{Q}_{\zeta}$ for every $C \subseteq \omega_{1}$; in particular, $A_{\xi}^{\prime} \in \mathbb{Q}_{\zeta}$ for every $\xi<\omega_{1}$; which is not so.

(c) If $\left\langle A_{n}\right\rangle_{n \in \mathbf{N}}$ is any sequence in $\mathscr{G}$, then (because $\left\langle Q_{\xi}\right\rangle_{\xi<\omega_{1}}$, and therefore $\left\langle\mathscr{G}_{\xi}\right\rangle_{\xi<\omega_{1}}$, are increasing) there is a $\xi<\omega_{1}$ such that every $A_{n}$ belongs to $\Phi_{\xi}$; an easy calculation shows that $\cup_{n \in \mathbf{N}} A_{n} \in \mathscr{G}_{\xi+2} \subseteq 9$. This shows that $\mathcal{G}$ is a $\sigma$-ideal of $\mathcal{P} I$, because of course any subset of a member of $\mathscr{G}$ belongs to $\mathscr{G}$. By (b), any uncountable disjoint family in $\mathscr{P} I$ meets $\mathscr{G}$; so that $\mathscr{G}$ is $\omega_{1}$-saturated. Observe that all singletons belong to 9 .

(d) Before completing the proof of (c), we deal with (d). By Lemma 7(d), the union of any $\omega_{1}$ members of 9 belongs to 9 . It follows at once (since the $\Phi_{\xi}$ increase with $\xi$ ) that $G=g_{\zeta}$ for some $\zeta<\omega_{1}$.

(c) concluded. Suppose, if possible, that there is a set $A \in \mathcal{P} I \backslash \mathcal{G}$ such that $A^{\bullet}$ is an atom in $\mathscr{P} I / \mathcal{G}$. Choose $A_{\xi}$, for $\xi<\omega_{1}$, as follows. $A_{0}=A$. Given that $A_{\xi} \notin \mathcal{G}$, choose $A_{\xi+1} \subseteq A_{\xi}$ such that $A_{\xi+1} \notin \mathbb{Q}_{\xi+\xi}$, where $\zeta$ is the ordinal of (d); then $A_{\xi+1} \notin 9$ so that $A \backslash A_{\xi+1} \in 9$. For limit ordinals $\xi>0$ set $A_{\xi}=\bigcap_{\eta<\xi} A_{\eta}$; then $A \backslash A_{\xi} \in \mathscr{9}$ because 9 is a $\sigma$-ideal, so $A_{\xi} \notin 9$. Continue.

Now $\mathcal{E}=\left\{A_{\omega_{1}}\right\} \cup\left\{A_{\xi} \backslash A_{\xi+1}: \xi<\omega_{1}\right\}$ is a disjoint family in $\mathscr{P} I$, so there is a $\theta<\omega_{1}$ such that $\cup \mathcal{E}^{\prime} \in \mathbb{Q}_{\theta}$ for every $\mathcal{E}^{\prime} \subseteq \mathcal{E}$ (by (a) above, since $\#(\mathcal{E}) \leqslant \omega_{1}$ ). But this implies that $A_{\xi} \in \mathbb{Q}_{\theta}$ for every $\xi<\omega_{1}$, which is not so. This completes the proof of (c).

10. Corollary. Let $X$ be a topological space. Let $\mathcal{C}$ be the collection of subsets of $X$ which are either open or closed, so that $\Re(\mathcal{C})$ is the algebra of Borel subsets of $X$. Let $\mathcal{E}$ be a point-finite $\mathscr{B}(\bigodot)$-additive family of subsets of $X$. Then there is a $\zeta<\omega_{1}$ such that $\varepsilon \subseteq \Re_{\zeta}(e)$.

Proof. In Theorem 9, take $I=\mathcal{E}, \mathbb{Q}_{\xi}=\left\{A: A \subseteq \mathcal{E}, \cup A \in \mathscr{B}_{\xi}(\bigodot)\right\}$. Because $\mathcal{E}$ is point-finite, $\left\langle\mathbb{Q}_{\xi}\right\rangle_{\xi<\omega_{1}}$ satisfies the conditions of Theorem 9. Take $\zeta<\omega_{1}$ such that $\mathscr{G}=\Phi_{\zeta}$; then $\{E\} \in \mathcal{G}=\Phi_{\zeta}$ for every $E \in \mathcal{E}$, i.e. $\mathcal{E} \subseteq \mathscr{B}_{\zeta}(\mathcal{C})$.

REMARK. This is in effect Theorem 1 of [7]. It was also found, independently, by B. S. Spahn [18]. For disjoint families $\mathcal{E}$, the result is covered by [13].

11. Lemma. Let $X$ and $Y$ be sets, and $\mathscr{Q} \subseteq \mathscr{P} X, D \subseteq X \times Y$.

(a) If $D \in \mathscr{B}_{\xi}(\{A \times C: A \in \mathbb{Q}, C \subseteq Y\})$ then every horizontal section of $D$ belongs to $\Re_{\xi}(Q)$.

(b) If every horizontal section of $D$ belongs to $\Re_{\xi}(\mathbb{Q})$, and $Q$ is countable, then $D \in \mathscr{G}_{2+\xi}(\{A \times C: A \in \mathbb{Q}, C \subseteq Y\})$. 
Proof. Induce on $\xi$. (Compare [1, Theorem 3].)

12. THEOREM. Let $\kappa$ be a cardinal. Then the following are equivalent:

(i) There is a separable metric space $X$ with $\mathcal{P} X=\mathscr{B}(X)$ and $\#(X)=\kappa$.

(ii) $\mathcal{P}_{\kappa}$ is countably generated (as $\sigma$-algebra).

(iii) $\mathscr{P}(\kappa \times J)=\mathscr{P}_{\kappa} \hat{\otimes}_{\sigma} \mathscr{P} J_{J}$ for every set $J$.

(iv) If $J$ is any set and $Q \subseteq \mathcal{P} J$ has cardinal $\leqslant \kappa$, there is a countable $\subseteq \subseteq \mathscr{P} J$ and a $\zeta<\omega_{1}$ such that $Q \subseteq \Re_{\zeta}(C)$.

Proof. (a) (i) $\Rightarrow$ (iii). Assume (i). If $\kappa$ is countable, the result is trivial. If $\kappa=\omega_{1}$, apply Theorem 9 with $I=\omega_{1}, Q_{\xi}=\Re_{\xi}(\mathcal{Q})$ where $\mathcal{U}$ is a countable base for a separable metrizable topology on $\omega_{1}$ for which every set is Borel. Then the ideal of Theorem 9 contains singletons and is closed under the unions of $\omega_{1}$ members (Lemma 7(d) again), so $\omega_{1} \in \mathcal{G}$, i.e. there is a $\zeta<\omega_{1}$ such that $\mathscr{P} \omega_{1}=\mathscr{B}_{\zeta}(\mathscr{Q})$. By Lemma 11(b) it follows that every subset of $\omega_{1} \times J$ belongs to $\mathscr{P} \omega_{1} \hat{\otimes}_{\sigma} \mathscr{P} J$, for any set $J$.

Now suppose that $\kappa>\omega_{1}$. In this case, if (i) is true of $\kappa$, it is surely true of $\omega_{1}$ as well; so that $\mathscr{P}\left(\omega_{1} \times \kappa\right)=\mathscr{P} \omega_{1} \hat{\otimes}_{\sigma} \mathscr{P}_{\kappa}$, by what we have just said. Once again, we apply Theorem 9 , this time with $I=\kappa, \mathbb{Q}_{\xi}=\mathscr{B}_{\xi}(\mathscr{U})$ where $\mathcal{Q}$ is a countable base for a separable metrizable topology on $\kappa$ for which every subset of $\kappa$ is Borel. But this time we can use (a) of Theorem 9 on the family of singletons of $\kappa$ to see that $\mathscr{P}_{\kappa} \subseteq \mathscr{B}_{\zeta}(\mathcal{Q})$ for some $\zeta<\omega_{1}$. So once again, by $11(\mathrm{~b})$, we have $\mathscr{P}(\kappa \times J)=\mathscr{P}_{\kappa}$ $\hat{\otimes}_{\sigma} \mathscr{P} J$ for every set $J$.

(b) (iii) $\Rightarrow$ (ii). Assume (iii), and take $J=\mathscr{P}_{\kappa}, D=\{(\xi, A): \xi \in A \subseteq \kappa\}$. As $D \in \mathcal{P}_{\kappa} \hat{\otimes}_{\sigma} \mathcal{P} J$, there are sequences $\left\langle A_{n}\right\rangle_{n \in \mathrm{N}}$ of subsets of $\kappa,\left\langle Q_{n}\right\rangle_{n \in \mathrm{N}}$ of subsets of $J$ such that

$$
D \in \mathscr{B}_{\zeta}\left(\left\{A_{m} \times \mathbb{Q}_{n}: m \in \mathbf{N}, n \in \mathbf{N}\right\}\right)
$$

for some $\zeta<\omega_{1}$. Take $\mathcal{C}=\left\{A_{n}: n \in \mathbf{N}\right\}$. Then every horizontal section of $D$ belongs to $\mathscr{B}_{\zeta}(\mathcal{C})$, by Lemma 11(a). But this shows that $\mathscr{P}_{\kappa}=\mathscr{B}_{\zeta}(\mathcal{C})$, so that $\mathcal{C}$ is a countable set generating $\mathscr{P}_{\kappa}$.

(c) (ii) $\Rightarrow$ (i). If $\mathcal{C}$ is a countable subset of $\mathscr{P}_{\kappa}$ which generates it as a $\sigma$-algebra, then the countable algebra of sets generated by $\mathcal{C}$ is a base for a separable metrizable topology on $\kappa$ for which every subset is Borel.

(d) (iii) $\Rightarrow$ (iv). Assume (iii), and let $Q \subseteq \mathcal{P} J$ have $\kappa$ or fewer members. Express $Q$ as $\left\{A_{\xi}: \xi<\kappa\right\}$. (We pass over the case $\mathbb{Q}=\varnothing$.) Set $A=\left\{(\xi, t): t \in A_{\xi}\right\} \subseteq \kappa \times J$. Then, as in (b) above, there must be a countable $\mathcal{C} \subseteq \mathcal{P} J_{\text {such }}$ that every vertical section of $A$ belongs to $\mathscr{B}_{\zeta}(\mathcal{C})$, for some $\zeta<\omega_{1}$; i.e. $\mathscr{Q} \subseteq \mathscr{B}_{\zeta}(\bigodot)$.

(e) (iv) $\Rightarrow$ (iii). Assume (iv), and let $A \subseteq \kappa \times J$. Let $\mathbb{Q}$ be the set of vertical sections

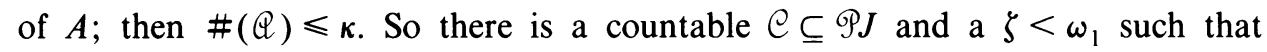
$Q \subseteq \Re_{\zeta}(\mathcal{C})$. By Lemma 11(b), on its side, we see that

$$
A \in \mathscr{P}_{2+\zeta}(\{B \times C: B \subseteq \kappa, C \in \mathcal{C}\}) \subseteq \mathscr{P}_{\kappa} \hat{\otimes}_{\sigma} \mathscr{P}_{J} .
$$

REMARKS. We observe that in (i), (ii) and (iii) there are equivalent forms such as

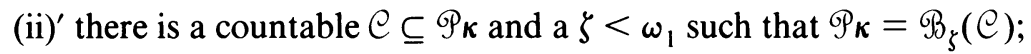

(iii) $)^{\prime}$ there is a $\zeta<\omega_{1}$ such that $\mathscr{P}(\kappa \times J)=\mathscr{B}_{\zeta}(\{B \times C: B \subseteq \kappa, C \subseteq J\})$ for any set $J$. 
It is not clear to us whether a reverse procedure can be applied to (iv); see Problem 23(f) below.

Note that $\mathrm{MA}(\kappa)$ implies (i) (see [11, p. 162]), and that (ii) implies that $2^{\kappa} \leqslant c$. Accordingly it is undecidable whether $\omega_{1}$, for instance, satisfies the conditions of this theorem. This is our reason for expressing (a) of Theorem 9 in its oddly conditional form; we do not know which $\kappa$ we shall be able to apply it to.

The equivalence of (ii) and (iii)', which is really the essence of this theorem (the rest being just repeated applications of Lemma 11), is given in [12, Theorem 48]; see also Corollary 20 below.

13. Corollary. Let $X$ be a set, $\mathbb{Q} \subseteq \mathcal{P} X, \mathcal{E} \subseteq \mathcal{P} X$ a point-finite $\mathscr{B}(\mathbb{Q})$-additive family. Then either $\mathcal{E}$ is boundedly $\Re(Q)$-additive or

(i) $\kappa_{d} \leqslant \#(\mathcal{E})$;

(ii) the continuum hypothesis is wildly false;

(iii) $\mathcal{P} \omega_{1}$ is not countably generated;

(iv) $\mathrm{MA}\left(\omega_{1}\right)$ is false.

Proof. Apply Theorem 9 with $I=\mathcal{E}, \mathbb{Q}_{\xi}=\left\{\mathcal{E}^{\prime}: \cup \mathcal{E}^{\prime} \in \mathscr{B}_{\xi}(\mathscr{Q})\right\}$ for $\xi<\omega_{1}$. If $I \in \mathscr{G}$, then $\mathscr{P} I=\mathscr{G}_{\zeta}$ for some $\zeta<\omega_{1}$, and $\mathcal{E}$ is $\mathscr{B}_{\zeta}(\mathscr{Q})$-additive. If $I \notin \mathscr{G}$, then $\mathscr{G}$ is a proper $\omega_{1}$-saturated $\sigma$-ideal of subsets of $\mathscr{G}$ such that $\mathscr{P} I / \mathscr{G}$ has no atoms, so that $\kappa_{d} \leqslant \#(I)=\#(\mathcal{E})$. Consequently, by Lemma 7(b), the continuum hypothesis is wildly false. At the same time, by (a) of Theorem 9 , we have $\mathscr{P}\left(\omega_{1} \times \#(\mathcal{E})\right) \neq \mathscr{P} \omega_{1}$ $\hat{\otimes}_{\sigma} \mathcal{P}\left(\#(\mathcal{E})\right.$ ), so (by Theorem 12) $\mathcal{P} \omega_{1}$ is not countably generated, and $\operatorname{MA}\left(\omega_{1}\right)$ must be false.

14. Corollary. Let $X$ be a topological space, $C_{\text {the }}$ set of subsets of $X$ which are either open or closed. Let $\mathcal{E} \subseteq \mathcal{P} X$ be a point-finite Borel-additive family of subsets of $X$. Then either $\mathcal{E}$ is boundedly $\mathscr{B}(\mathcal{C})$-additive or

(i) $\kappa_{d} \leqslant \#(\mathcal{E})$; the continuum hypothesis is wildly false;

(ii) $\mathcal{P} \omega_{1}$ is not countably generated; $\operatorname{MA}\left(\omega_{1}\right)$ is false;

(iii) every second-countable subset of $X$ meets only countably many members of $\mathcal{E}$.

Proof. Take $\mathcal{A}=\bigodot$ in Corollary 13. If $\mathcal{E}$ is not $\mathscr{B}_{\zeta}(\bigodot)$-additve for any $\zeta<\omega_{1}$, then (i) and (ii) must be true. But now suppose, if possible, that $Y \subseteq X$ were a second-countable subspace meeting uncountably many members of $\mathcal{E}$. By Lemma 5 , there is a $Z \subseteq Y$, with \#(Z)= $\omega_{1}$, and a $\mathcal{H} \subseteq \mathcal{E}$ such that each member of $Z$ belongs to just one member of $\mathcal{H}$. Now every subset of $Z$ is of the form $Z \cap \cup \mathcal{H}^{\prime}$ for some $\mathcal{H}^{\prime} \subseteq \mathcal{H}$, so $\mathscr{P} Z=\mathscr{B}(Z)$. But also $Z$ is second-countable, so that $\mathscr{B}(Z)$ is countably generated and $\mathscr{P} Z$ is countably generated, contradicting (ii).

15. LemMa. Let $X$ be a metric space, $\mathcal{E}$ a point-finite Borel-additive family of subsets of $X$. Then the following are equivalent:

(i) $\mathcal{E}$ is boundedly Borel-additive;

(ii) if $\mathscr{U}$ is a discrete family of open sets in $X$ and $S \subseteq \mathscr{U} \times \mathcal{E}$ then $G_{S}=$ $\cup_{(U, E) \in S} E \cap U$ is Borel;

(iii) whenever $\left\langle U_{\xi}\right\rangle_{\xi<\omega_{1}}$ is a discrete family of open sets in $X$ and $\left\langle\mathcal{E}_{\xi}\right\rangle_{\xi<\omega_{1}}$ is a family of subsets of $\mathcal{E}$, there is a $\xi<\omega_{1}$ such that $U_{\xi} \cap \cup \mathcal{E}_{\xi} \in \mathscr{B}_{\xi}(X)$. 
Proof. (a) (i) $\Rightarrow$ (ii). Suppose that $\mathscr{E}$ is $\mathscr{B}_{\zeta}$-additive, and that $\mathscr{Q}$ is a discrete family

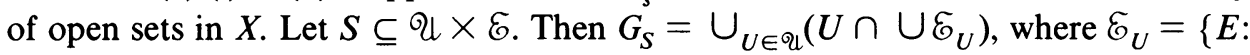
$(U, E) \in S\}$. But $\cup \mathcal{E}_{U} \in \mathscr{B}_{\zeta}(X)$ for every $U \in \mathcal{Q}$, so $G_{S} \in \mathscr{B}_{\zeta}(X)$, by Theorem 3(b).

(b) (ii) $\Rightarrow$ (iii). Assume (ii). Given $U_{\xi}, \mathcal{E}_{\xi}$ set

$$
S=\left\{\left(U_{\xi}, E\right): \xi<\omega_{1}, E \in \mathcal{E}_{\xi}\right\} .
$$

Then $G_{S} \in \mathscr{B}_{\xi}(X)$ for some $\xi$, so $U_{\xi} \cap \cup \varepsilon_{\xi}=U_{\xi} \cap G_{S} \in \mathscr{B}_{\xi}(X)$.

(c) (iii) $\Rightarrow$ (i). Note first that if any separable subset of $X$ meets uncountably many members of $\mathcal{E}$, then the result follows from Corollary 14(iii). So let us suppose that every separable subset of $X$ meets only countably many members of $\mathcal{E}$.

Observe next that if $\mathscr{U}$ is any discrete family of open sets in $X$, then there must be a $\xi<\omega_{1}$ such that

$$
\left\{U: U \in \mathcal{Q}, \exists \mathcal{E}^{\prime} \subseteq \mathcal{E}, U \cap \cup \mathcal{E}^{\prime} \notin \mathscr{B}_{\xi}(X)\right\}
$$

is countable; for otherwise we could choose inductively $U_{\xi} \in \mathscr{Q}$ and $\mathcal{E}_{\xi} \subseteq \mathcal{E}$ to contradict hypothesis (iii). Let $\left\langle\mathcal{U}_{n}\right\rangle_{n \in \mathbf{N}}$ be a sequence of discrete families of open sets in $X$ such that $\cup_{n \in N} \mathscr{Q}_{n}$ is a base for the topology of $X$. Then there is a $\xi<\omega_{1}$ such that

$$
\forall n \in \mathbf{N}, \quad \mathscr{V}_{n}=\left\{U: U \in \mathcal{Q}_{n}, \exists \mathcal{E}^{\prime} \subseteq \mathcal{E}, U \cap \cup \mathcal{E}^{\prime} \notin \mathscr{B}_{\xi}(X)\right\}
$$

is countable. Set $\mathscr{U}_{n}^{*}=\mathcal{Q}_{n} \backslash \mathcal{V}_{n}$ for each $n \in \mathbf{N}$, and

$$
Y=\bigcup_{n \in \mathbf{N}}\left(\cup \mathscr{Q}_{n}^{*}\right), \quad Z=X \backslash Y .
$$

As $\left\{Z \cap U: U \in \cup_{n \in \mathbf{N}} \mathcal{T}_{n}\right\}=\left\{Z \cap U: U \in \cup_{n \in \mathbf{N}} \mathscr{Q}_{n}\right\}$ is a base for the topology of $Z, Z$ is separable, and $\mathcal{E}_{0}=\{E: E \in \mathcal{E}, E \cap Z \neq \varnothing\}$ is countable. Let $\eta<\omega_{1}$ be such that $\mathcal{E}_{0} \subseteq \mathscr{B}_{\eta}(X)$.

Try $\zeta=\max (\xi, \eta)+1$. If $\mathcal{E}^{\prime} \subseteq \mathcal{E}$, then for each $n \in \mathbf{N}$,

$$
U \cap \cup \xi^{\prime} \in \mathscr{B}_{\xi}(X) \quad \forall U \in \mathcal{Q}_{n}^{*}
$$

so (as $\mathscr{U}_{n}^{*}$ is discrete) $\cup \mathscr{U}_{n}^{*} \cap \cup \mathcal{E}^{\prime} \in \mathscr{B}_{\xi}(X)$ and

$$
Y \cap \cup \mathcal{E}^{\prime}=\bigcup_{n \in \mathbf{N}}\left(\cup \mathcal{U}_{n}^{*} \cap \cup \mathcal{E}^{\prime}\right) \in \mathscr{B}_{\xi+1}(X) .
$$

On the other hand, $Z \cap \cup \mathcal{E}^{\prime}=Z \cap \cup\left(\mathcal{E}^{\prime} \cap \varepsilon_{0}\right) \in \mathscr{B}_{\eta+1}(X)$. So

$$
\cup \mathcal{E}^{\prime}=\left(Y \cap \cup \mathcal{E}^{\prime}\right) \cup\left(Z \cap \cup \mathcal{E}^{\prime}\right) \in \mathscr{B}_{\zeta}(X) .
$$

As $\mathcal{E}^{\prime}$ is arbitrary, $\mathcal{E}$ is again boundedly Borel-additive.

16. Theorem. Let $X$ and $Y$ be metric spaces, $R \subseteq X \times Y$ a lower Borel measurable relation with compact vertical sections. Then either $R$ is of bounded class or

(i) $Y$ has a discrete subset of cardinal $\kappa_{d}$;

(ii) the continuum hypothesis is wildly false;

(iii) $\mathcal{P} \omega_{1}$ is not countably generated; $\operatorname{MA}\left(\omega_{1}\right)$ is false;

(iv) $X$ has a discrete subset of cardinal $\kappa_{d}$;

(v) $R[Z]$ is separable for every separable $Z \subseteq X$; 
(vi) there is a $u \in Y$ such that $R^{-1}[H]$ is nonseparable for every neighbourhood $H$ of $u$;

(vii) $X$ is not absolutely analytic (i.e. is not a Souslin-Fु subset of its completion);

(viii) Fleissner's Proposition $\mathrm{P}$ is false; the Product Measure Extension Axiom is false.

Definitions. Fleissner's Proposition P states: if $X$ is a metric space, then every point-finite Borel-additive family of subsets of $X$ is $\sigma$-discretely decomposable. The Product Measure Extension Axiom states: if $\mu$ is the usual power measure on $\{0,1\}^{\kappa}$, where $\kappa$ is any cardinal, then $\mu$ has an extension $\bar{\mu}$ defined on every subset of $\{0,1\}^{\kappa}$ such that $\bar{\mu}$ is $c$-additive, i.e. the union of fewer than $c$ negligible sets is always negligible. In [4] some of the many remarkable consequences of Proposition $\mathrm{P}$ are given. In [5] there is a proof that the Product Measure Extension Axiom implies Proposition P.

The Product Measure Extension Axiom is of course even less plausible than the existence of a real-valued measurable cardinal; but $\mathrm{K}$. Kunen has shown that if it consistent to assume that there is a strongly compact cardinal, then it is consistent to assume the Product Measure Extension Axiom; so that we have some grounds for hoping that it is consistent.

Observe that Proposition P implies that any separable metric space in which every subset is Borel must be countable; by Theorem 12, if follows that $\mathcal{P} \omega_{1}$ cannot be countably generated.

Proof OF Theorem 16. (a) Examining the proof of Proposition 8(b), we see that if $R$ is not of bounded class then there is a discrete family $\mathscr{U}$ of open sets in $Y$ such that $\mathcal{E}=\left\{R^{-1}[U]: U \in \mathscr{Q}\right\}$ is a point-finite, Borel-additive family which is not boundedly Borel-additive. By Corollary $14, \kappa_{d} \leqslant \#(\mathcal{E}) \leqslant \#(\mathcal{U})$; this proves (i); also (ii) and (iii) follow from Corollary 14.

(b) For (iv), suppose, if possible, that every discrete set in $X$ had cardinal $<\kappa_{d}$. Then there is a base $\mathscr{V}$ for the topology of $X$ with $\#(\mathscr{V})<\kappa_{d}\left(\right.$ since $\left.\operatorname{cf}\left(\kappa_{d}\right)>\omega\right)$. At the same time, since $\#(\mathcal{E}) \geqslant \kappa_{d}$ and $\mathcal{E}$ is a point-finite, there is a $Z \subseteq X$ and an $\mathcal{E}_{1} \subseteq \mathcal{E}$ such that $\#(Z)=\kappa_{d}$ and each member of $Z$ belongs to just one member of $\mathcal{E}_{1}$. Let $\mathscr{G}$ be a proper $\omega_{1}$-saturated $\sigma$-ideal of $\mathscr{P} Z$ such that $\mathscr{P} Z / \mathcal{G}$ is atomless; then all singletons must belong to 9 . By Lemma 7(c),

$$
W=\cup\{V \cap Z: V \in \mathscr{V}, V \cap Z \in \mathscr{T}\} \in \mathscr{q},
$$

and $Z \backslash W \notin \mathscr{G}$. But now observe that because $\mathcal{G}$ is $\omega_{1}$-saturated, and $(Z \backslash W) \cap V \notin \Phi$ whenever $V \in \mathcal{V}$ and $(Z \backslash W) \cap V \neq \varnothing, Z \backslash W$ must satisfy the countable chain condition. So $Z \backslash W$ is separable. But of course $Z \backslash W$ is uncountable, so meets uncountably many members of $\mathcal{E}_{1} \subseteq \mathcal{E}$; contradicting Corollary 14(iii).

(c) For (v), suppose that $Z \subseteq X$ and that $R[Z]$ is not separable. Let $\varepsilon>0$ be such that $R[Z]$ cannot be covered by countably many sets of diameter $\leqslant \varepsilon$, and for each $n \in \mathbf{N}$ let $\mathcal{Q}_{n}$ be a locally finite cover of $Y$ by open sets of diameter $\leqslant 2^{-n} \varepsilon$, so that $\cup_{n \in \mathbf{N}}{ }^{2}{ }_{n}$ is a base for the topology of $Y$. Then we see that any compact subset of $Y$ can meet only finitely many members of any $\mathscr{Q}_{n}$, so that (just as in Proposition 8(b)) $\varepsilon_{n}=\left\{R^{-1}[U]: U \in \mathscr{Q}_{n}\right\}$ is point-finite for each $n \in \mathbf{N}$, and there is some $m \in \mathbf{N}$ 
such that $\varepsilon_{m}$ is not boundedly Borel-additive. At the same time, $R[Z]$ is covered by $\mathcal{U}_{m}$, but not covered by any countable subset of $\mathscr{U}_{m}$; so that $Z$ meets uncountably many members of $\varepsilon_{m}$, and by 14(iii) again cannot be separable.

(d) For (vi), suppose, if possible, that

$$
\mathcal{H}=\left\{H: H \subseteq Y \text { open, } R^{-1}[H] \text { is separable }\right\}
$$

covers $Y$. Then every $\mathscr{U}_{n}$ in Proposition $8(\mathrm{~b})$ can be taken to be a refinement of $\mathcal{H}$, and the $\mathscr{U}$ of (a) above will be a refinement of $\mathcal{H}$, so that every member of $\mathcal{E}$ is separable. By Lemma 15 there is a discrete family $\left\langle V_{\xi}\right\rangle_{\xi<\omega_{1}}$ of open sets in $X$ and a family $\left\langle\mathcal{E}_{\xi}\right\rangle_{\xi<\omega_{1}}$ of subsets of $\mathcal{E}$ such that $V_{\xi} \cap \cup \mathcal{E}_{\xi} \notin \mathscr{B}_{\xi}(X)$ for every $\xi<\omega_{1}$. For each $E \in \mathcal{E}, I(E)=\left\{\xi: E \in \mathcal{E}_{\xi}, E \cap V_{\xi} \neq \varnothing\right\}$ is countable; enumerate $I(E)$ as $\langle\theta(E, n)\rangle_{n \in J(E)}$ where $J(E) \subseteq \mathbf{N}$. Set

$$
\mathcal{E}_{n \xi}=\{E: E \in \mathcal{E}, n \in J(E), \theta(E, n)=\xi\},
$$

and $E_{n \xi}=\cup \mathcal{E}_{n \xi}$. For fixed $n,\left\langle\mathcal{E}_{n \xi}\right\rangle_{\xi<\omega_{1}}$ is a disjoint family of subsets of $\mathcal{E}$, so that $\left\langle E_{n \xi}\right\rangle_{\xi<\omega_{1}}$ is point-finite and Borel additive. By Corollary 10, there is a $\zeta(n)<\omega_{1}$ such that every $E_{n \xi}$ belongs to $\mathscr{B}_{\zeta(n)}(X)$. Take $\zeta=\sup _{n \in \mathrm{N}}(\zeta(n)+1)$. Then

$$
\begin{aligned}
V_{\zeta} \cap \cup \mathcal{E}_{\zeta} & =V_{\zeta} \cap \cup\{E: E \in \mathcal{E}, \zeta \in I(E)\} \\
& =V_{\zeta} \cap \bigcup_{n \in \mathbf{N}} \bigcup\{E \in \mathcal{E}, n \in J(E), \theta(E, n)=\zeta\} \\
& =V_{\zeta} \cap \bigcup_{n \in \mathbf{N}} E_{n \zeta} \in \Re_{\zeta}(X),
\end{aligned}
$$

contrary to the choice of $V_{\zeta}$ and $\varepsilon_{\zeta}$.

(e) For (vii) and (viii), observe that $\mathcal{E}$ cannot be $\sigma$-discretely decomposable; because by Corollary 10 there is a $\xi<\omega_{1}$ such that $\mathcal{E} \subseteq \Re_{\xi}(X)$, and if $\mathcal{E}$ were $\sigma$-discretely decomposable it would follow that $\mathcal{E}$ was $\Re_{\xi+1}(X)$-additive, by $[4,2.7]$ (or Theorem 3(b) above). By [8, Theorem 1], it follows that $X$ cannot be absolutely analytic. Also, of course, Proposition $\mathrm{P}$ and the Product Measure Extension Axiom must be false.

REMARK. (vii) above was found independently by B. S. Spahn [18].

17. Corollary. Let $X$ and $Y$ be metric spaces, and $f: X \rightarrow Y$ a Borel measurable function. Then either $f$ is of bounded class or (i)-(viii) of Theorem 16 are all true (reading $f[Z], f^{-1}[H]$ for $R[Z], R^{-1}[H]$ in (v) and (vi)).

18. Proposition. Suppose $X$ is a metric space and $\mathcal{E}$ is a point-finite Borel-additive family of subsets of $X$ which is not boundedly Borel-additive. Then there is a similar family $\mathscr{F}$ of subsets of $X$ with $\#(\mathscr{F}) \leqslant c$.

Proof. In Theorem 9 take

$$
\begin{gathered}
I=\mathcal{E}, \quad \mathscr{Q}_{\xi}=\left\{\mathcal{E}^{\prime}: \mathcal{E}^{\prime} \subseteq \mathcal{E}, \cup \mathcal{E}^{\prime} \in \mathscr{B}_{\xi}(X)\right\}, \\
\Phi_{\xi}=\left\{\mathcal{E}^{\prime}: \mathcal{E}^{\prime} \subseteq \mathcal{E}, \mathscr{P} \mathcal{E}^{\prime} \subseteq \mathbb{Q}_{\xi}\right\}, \quad g=\bigcup_{\xi<\omega_{1}} \Phi_{\xi},
\end{gathered}
$$


and $\zeta<\omega_{1}$ such that $G=\Phi_{\zeta}$, as in Theorem 9(d). By Lemma 7(a), there is a family $\left\langle\mathcal{E}_{\xi}\right\rangle_{\xi<c}$ in 9 such that $\mathscr{E}=\cup_{\xi<c} \mathcal{E}_{\xi}$; we can take $\left\langle\mathcal{E}_{\xi}\right\rangle_{\xi<c}$ to be disjoint. Set $F_{\xi}=\cup \mathcal{E}_{\xi}, \mathscr{F}=\left\{F_{\xi}: \xi<\mathfrak{c}\right\}$. Then $\mathscr{F}$ is point-finite and Borel-additive.

Suppose, if possible, that $\mathscr{F}$ is $\mathscr{B}_{\eta}(X)$-additive for some $\eta$. In this case, for any $\mathcal{E}^{\prime} \subseteq \mathcal{E}$, we have

$$
\mathcal{E}^{\prime} \cap \varepsilon_{\xi} \in \mathscr{q}=g_{\xi} \quad \forall \xi<c
$$

so that $E_{\xi}=\cup\left(\mathcal{E}^{\prime} \cap \varepsilon_{\xi}\right) \in \mathscr{B}_{\xi}(X)$ for every $\xi$. Then

$$
\cup \mathcal{E}^{\prime}=\bigcup_{\xi<c} E_{\xi}=\bigcup_{\xi<c} E_{\xi} \cap F_{\xi} \in \Re_{\eta+1+\xi}(X)
$$

by Theorem $3\left(\right.$ b). So $\mathcal{E}$ is $\mathscr{B}_{\eta+1+\zeta}(X)$-additive, contrary to hypothesis.

Thus $\mathscr{F}$ is the required family.

19. THEOREM. Let $X$ and $Y$ be metric spaces and $f: X \rightarrow Y$ a Borel isomorphism. Then $f$ and $f^{-1}$ are of bounded class.

REMARK. In the language of [10], $f$ is a "generalized homeomorphism".

Proof. As in the proof of Proposition 8(a), it will be enough to show that if $Q$ is a discrete collection of open sets in $Y$, then $\mathcal{E}=\left\{f^{-1}[U]: U \in \mathcal{U}\right\}$ is boundedly Borel-additive. We use the criterion of Lemma 15(iii). If $\left\langle V_{\xi}\right\rangle_{\xi<\omega_{1}}$ is a discrete family of open sets in $X$ and $\left\langle\varepsilon_{\xi}\right\rangle_{\xi<\omega_{1}}$ is any family of subsets of $\mathcal{E}$, express each $\mathcal{E}_{\xi}$ as $\left\{f^{-1}[U]: U \in \mathcal{Q}_{\xi}\right\}$ where $\mathscr{Q}_{\xi} \subseteq \mathcal{U}$. Consider $G=\cup_{\xi<\omega_{1}} V_{\xi} \cap \cup \mathcal{E}_{\xi}$. Then

$$
\begin{aligned}
f[G] & =\bigcup_{\xi<\omega_{1}} f\left[V_{\xi} \cap \cup \mathcal{E}_{\xi}\right]=\bigcup_{\xi<\omega_{1}} f\left[V_{\xi}\right] \cap \cup \mathscr{Q}_{\xi} \\
& =\bigcup_{U \in \mathcal{U}}\left(U \cap \cup\left\{f\left[V_{\xi}\right]: \xi<\omega_{1}, U \in \mathcal{Q}_{\xi}\right\}\right) .
\end{aligned}
$$

But examine $\mathscr{F}=\left\{f\left[V_{\xi}\right]: \xi<\omega_{1}\right\}$. $\mathscr{F}$ is a point-finite family of subsets of $Y$ which is Borel-additive because $f^{-1}$ is Borel measurable, and $\#(\mathscr{F}) \leqslant \omega_{1}<\kappa_{d}$. So by Corollary 13 there is an $\eta<\omega_{1}$ such that $\mathscr{F}$ is $\mathscr{B}_{\eta}(Y)$-additive. In this case, $\cup\left\{f\left[V_{\xi}\right]\right.$ : $\left.\xi<\omega_{1}, U \in \mathcal{Q}_{\xi}\right\} \in \mathscr{B}_{\eta}(Y)$ for every $U \in \mathcal{Q}$, so $f[G] \in \mathscr{B}_{\eta}(Y)$ and $f[G]$ is Borel. Returning to $X$, we see that $G=f^{-1}[f[G]]$ is Borel; say $G \in \mathscr{B}_{\xi}(X)$. Then

$$
V_{\xi} \cap \cup \mathcal{E}_{\xi}=G \cap V_{\xi} \in \mathscr{B}_{\xi}(X) .
$$

As $\left\langle V_{\xi}\right\rangle_{\xi<\omega_{1}}$ and $\left\langle\mathcal{E}_{\xi}\right\rangle_{\xi<\omega_{1}}$ are arbitrary, $\mathcal{E}$ is boundedly Borel-additive, as required.

REMARK. This answers a question of A. H. Stone [16].

20. Corollary. If $X$ is a metric space such that every subset is Borel, then the Borel classes of the subsets of $X$ are bounded.

Proof. Apply Theorem 19 with $Y$ a discrete space with the same cardinality as $X$.

REMARK. This is Theorem 48 of [12].

21. Corollary. Let $X$ and $Y$ be metric spaces such that $\mathscr{B}(X)=\mathscr{\rho} X$ and $\Re(Y)=\mathscr{P} Y$. Then $\Re(X \times Y)=\mathscr{P}(X \times Y)$.

Proof (CF. [6, Corollary 3.7]). We have $\xi, \eta<\omega_{1}$ such that $\mathscr{P} X=\mathscr{G}_{\xi}(X)$, $\mathscr{P} Y=\mathscr{B}_{\eta}(Y)$. Let $\mathcal{E}$ be $\left\{\pi_{1}^{-1}[\{t\}]: t \in X\right\} \subseteq \mathcal{P}(X \times Y)$. Then $\mathcal{E}$ is a disjoint family 
which is $\mathscr{B}_{\xi}(X \times Y)$-additive. Let $A$ be any subset of $X \times Y$. Then $A$ is expressible as $\cup_{t \in X} \pi_{1}^{-1}[\{t\}] \cap A_{t}$ where $A_{t}=\{(s, u): s \in X, u \in Y,(t, u) \in A\} \in \Re_{\eta}(X \times Y)$. By Theorem 3(b), $A \in \mathscr{B}_{\xi+1+\eta}(X \times Y)$. As $A$ is arbitrary, $\mathscr{B}(X \times Y)=\mathscr{P}(X \times Y)$.

22. An independence result. The defect of $\S \S 13,14,16$ and 17 above is that we do not know whether it is ever possible for the second alternatives to hold; we think it quite likely, indeed, that there is a proof in ZFC that every Borel measurable function between metric spaces is of bounded class. If so, however, the proof is unlikely to come from a corresponding strengthening of Theorem 9, because of the following.

PROPOSITION. If one of the following statements is relatively consistent with ZFC, so is the other:

(A) there is a two-valued-measurable cardinal;

(B) there is a set I and a family $\left\langle Q_{\xi}\right\rangle_{\xi<\omega_{1}}$ as in Theorem 9, with I $\notin 9$.

Proof. (a) Suppose that (B) is consistent. Then by [15, Theorem 6] (A) is consistent.

(b) Suppose that (A) is consistent. Following through the argument of [15, §4], we see that if we start with a two-valued-measurable cardinal $\kappa$ we obtain a Booleanvalued model in which $\mathfrak{c}$ carries a measure $\mu$, defined on every subset of $\mathfrak{c}$, for which there is a family of $c$ independent sets. Consequently (in the new model) there must be a probability space $(X, \mathscr{P} X, \nu)$ which is Maharam homogeneous of uncountable type $\lambda$. Let $\left\langle E_{\xi}\right\rangle_{\xi<\lambda}$ be a generating family of independent sets of measure $\frac{1}{2}$. Write $Q_{\xi}$ for the $\sigma$-subalgebra of $\mathscr{P} X$ generated by

$$
\{E: \nu E=0\} \cup\left\{E_{\eta}: \eta<\xi \text { or } \eta \geqslant \omega_{1}\right\} .
$$

Then $E_{\xi} \notin \mathbb{Q}_{\xi}$ so that $\left\langle\mathbb{Q}_{\xi}\right\rangle_{\xi<\omega_{1}}$ is a strictly increasing family of $\sigma$-algebras with union $\mathcal{P} X$, giving an example for (B).

23. Concluding remarks. (a) The following fundamental problem, due to A. H. Stone, is left open by the work above: If $X$ and $Y$ are metric spaces and $f: X \rightarrow Y$ is a Borel measurable function, does $f$ have to be of bounded class [17, p. 245] ${ }^{1}$ From Corollary 17 we see that under any one of a varied assortment of special axioms the answer is "yes", and we suspect that this may be true in ZFC alone. Note that by Proposition 8(a) it is enough to consider the case in which $Y$ is discrete; by Corollary

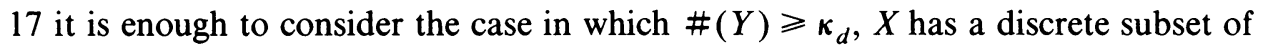
cardinal $\kappa_{d}, f[Z]$ is countable for every separable $Z \subseteq X$, and $f^{-1}[\{u\}]$ is nonseparable for every $u \in Y$; and by Proposition 18 it is enough to consider the case $\#(Y) \leqslant c$. (An examination of the arguments of [5] shows that now something less than the full Product Measure Extension Axiom is enough to clear things up; we need only suppose the existence of a countably-additive measure defined on all subsets of $\{0,1\}^{\mathrm{c}}$ which extends the usual measure. In consistency terms this is much

\footnotetext{
'In a review of this paper (MR 51-843) the second author incorrectly stated that this problem had been solved.
} 
weaker than the Product Measure Extension Axiom, being in fact equiconsistent with the existence of a two-valued measurable cardinal.)

(b) In [6, Corollary 3.7] the second author has shown that if $X, Y$ and $Z$ are metric spaces and $f: X \rightarrow Y$ and $g: X \rightarrow Z$ are Borel measurable functions of bounded class, then the function $t \mapsto(f t, g t): X \rightarrow Y \times Z$ is Borel measurable (and of bounded class). On the other hand, if $f: X \rightarrow Y$ is a function such that

$$
t \mapsto(t, f t): X \rightarrow X \times Y
$$

is Borel measurable, then $f$ is of bounded class, because $t \mapsto(t, f t)$ is a Borel isomorphism between $X$ and the graph of $f$, and we can use Theorem 19. So we see that Stone's question is equivalent to: If $X, Y$ and $Z$ are metric spaces and $f: X \rightarrow Y$, $g: X \rightarrow Y$ are Borel functions, does $t \mapsto(f t, g t)$ have to be Borel measurable?

(c) In the work above we have generally spoken of point-finite families of sets rather than disjoint families, and it is perhaps surprising that it makes so little difference. A reason appears in the following result of B. S. Spahn. Let $X$ be a set, $\Sigma$ a $\sigma$-algebra of subsets of $X$, and $\mathscr{F}$ a point-finite $\Sigma$-additive collection of sets such that $\#(\mathscr{F}) \leqslant c$. Then there is a family $\left\langle H_{F}^{k}\right\rangle_{F \in \mathscr{F}, k \in N}$ of sets such that (i) $\left\langle H_{F}^{k}\right\rangle_{F \in \mathscr{F}}$ is disjoint and $\Sigma$-additive for each $k \in \mathbf{N}$ and (ii) $\cup_{k \in \mathbf{N}} H_{F}^{k}=F$ for each $F \in \mathscr{F}$. In view of Proposition 18, it follows at once that if $X$ is a metric space and $\mathcal{E}$ is a point-finite Borel-additive family of subsets of $X$ which is not boundedly Borel-additive, then there is a similar disjoint family $\mathscr{K}$ with $\#(\mathcal{H}) \leqslant c$. In particular, the conditions (a) and (b) of Proposition 8 are equivalent. So we see that another form of Stone's question is: If $X$ and $Y$ are metric spaces and $R \subseteq X \times Y$ is a lower Borel measurable relation with compact vertical sections, does $R$ have to be of bounded class?

(d) Again in [6], it is shown that if $R \subseteq X \times Y$ is a lower Borel measurable relation of bounded class with compact vertical sections, it has a Borel measurable selector (of bounded class). So we are led to the question: If $X$ and $Y$ are metric spaces and $R \subseteq X \times Y$ is a lower Borel measurable relation with compact vertical sections, does $R$ have to have a Borel measurable selector? We do not know whether this is equivalent to Stone's question.

(e) A question of lesser importance is whether there are any results of the type considered in this paper for functions $f: X \rightarrow Y$ where $X$ is metrizable and $Y$ is a compact Hausdorff space. For instance, if $Y$ is a compact Hausdorff space, is every Borel measurable $f:[0,1] \rightarrow Y$ of bounded class?

(f) Finally, a question which arises out of Theorem 12 is: Are the four conditions there equivalent to

(iv)' if $J$ is any set and $Q \subseteq \mathcal{P} J$ has cardinal $\leqslant \kappa$, there is a countable $\mathcal{C} \subseteq \mathcal{P} J$ such that $Q \subseteq \Re(C)$ ?

\section{REFERENCES}

1. R. H. Bing, W. W. Bledsoe and R. D. Mauldin, Sets generated by rectangles, Pacific J. Math. 51 (1974), 27-36.

2. W. W. Comfort and S. Negrepontis, The theory of ultrafilters. Springer-Verlag, Berlin and New York, 1974.

3. F. Drake, Set theory, North-Holland, Amsterdam, 1974. 
4. W. G. Fleissner, An axiom for nonseparable Borel theory, Trans. Amer. Math. Soc. 251 (1979), 309-328.

5. W. G. Fleissner, R. W. Hansell and H. J. K. Junnila, PMEA implies Proposition P, Topology Appl. 13 (1982), 255-262.

6. R. W. Hansell, Borel-additive families and Borel maps in metric spaces, General Topology and Modern Analysis (L. F. McAuley and M. M. Rao, eds.), Academic Press, New York, 1981, pp. 405-416.

7. __ Point-finite Borel-additive families are of bounded class, Proc. Amer. Math. Soc. 83 (1981), $375-378$.

8. J. Kaniewski and R. Pol, Borel-measure selectors for compact-valued mappings in the non-separable case, Bull. Acad. Polon. Sci. Sér. Math. Astronom. Phys. 23 (1975), 1043-1050.

9. K. Kunen, Inaccessibility properties of cardinals, Ph. D. dissertation, Stanford Univ., 1968.

10. K. Kuratowski, Topology.I, Academic Press, New York, 1966.

11. D. A. Martin and R. M. Solovay, Internal Cohen extensions, Ann. Math. Logic 2 (1970), 143-178.

12. A. W. Miller, On the length of Borel hierarchies, Ann. Math. Logic 16 (1979), 233-267.

13. D. Preiss, Completely additive disjoint system of Baire sets is of bounded class, Comment. Math. Univ. Carolin. 15 (1974), 341-344.

14. B. V. Rao, On discrete Borel spaces and projective sets, Bull. Amer. Math. Soc. 75 (1969), 614-617.

15. R. M. Solovay, Real-valued measurable cardinals, Proc. Sympos. Pure Math., vol. 12, Amer. Math. Soc., Providence, R.I., 1971, pp. 397-428.

16. A. H. Stone, Non-separable Borel sets, Rozprawy Math. 28 (1962).

17. __ Some problems of measurability (Topology Conf., Blacksburg, Virginia), Lecture Notes, in Math., vol. 375, Springer-Verlag, Berlin and New York, 1974.

18. B. S. Spahn, Thesis, Warsaw, 1981.

Department of Mathematics, University of EsSex, Colchester, England

Department of Mathematics, University of Connecticut, Storrs, Connecticut 06268

Department of Mathematics, University of Helsinki, Helsinki, Finland 